\title{
Communicatiom
}

\section{Towards road safety in LMICs: vehicles that guide drivers on self-explaining roads}

\section{Hans Godthelp}

1 Road safety for all, Voorburg, The Netherlands, hg@roadsafetyforall.

2 Correspondence: hg@roadsafetyforall.org

Received: date; Accepted: date; Published: date

\begin{abstract}
:
Traffic collisions cause a huge problem of public health in low and middle income countries.. The safe system approach is generally considered as the leading concept on the way to road safety. Based on the fundamental premise that humans make mistakes, the overall traffic system should be 'forgiving'. Sustainable safe road design is one of the key elements of the safe system approach. However, the road design principles behind the safe system approach are certainly not leading in today's infrastructure developments in most LMICs. Cities are getting larger and road networks are expanding. In many cases, existing through-roads in local communities are up-graded, resulting in heavy traffic loads and high speeds on places, that are absolutely not suited for this kind of through-traffic. Furthermore a safe system would require that functional design properties of cars and roads would be conceptually integrated, which is not the case at all. Although advanced driver assistance systems are on their way of development for quite a long period, their potential role in the safe system concept is mostly unclear and at least strongly underexposed. The vision on future cars is dominated by the concept of automation. This paper argues that the way to self-driving cars, should take a route via the concept of guidance, i.e. vehicles that guide drivers, both on self-explaining roads and on more or less unsafe roads. Such an in-vehicle support system may help drivers to choose transport mode, route and speed, based on criteria related to safety and sustainability. It is suggested to develop a driver assistance system using relatively simple and cheap technologies, particularly for the purpose of use in LMICs. Such a GUIDE (Generic User Interface for Driving Evolution) may make roads self-explaining - not only by their physical design characteristics - but also by providing in-car guidance for drivers. In future the functional characteristics of both cars and roads should be conceptualized into one integrated safe system, in which the user plays the central role. As such GUIDE may serve as the conceptual bridge between vehicle and roadway characteristics. It is argued that GUIDE is necessary to bring a breakthrough in road safety developments in LMICs and also give an acceleration towards zero fatalities in HICs.
\end{abstract}

Keywords: 1; road safety, 2; advanced driver assistance, 3 safe system approach, 4 LMIC.

\section{Introduction}

The number of traffic fatalities in The Netherlands reached a peak value of 3264 in 1972. After that year a long and steady period of safety programs followed Passive safety measures reduced the consequences of accidents and obligatory seat belt use may be considered as one of the most successful in this category. Strategies for law enforcement followed, not only to increase seat belt use but also to improve speed behavior and reduce alcohol use. All of these measures gave a first boost towards a reduction of the number of fatalities. A more or less definite breakthrough was 
reached by developing the so-called concept of Sustainable safety, i.e. the notion that the traffic system should be resistant to human errors. Fig. 1 gives the development of the road safety figures over the years with a lowest number of 570 in 2014. Developments similar to The Netherland took place in most of the other Western European countries and internationally the underlying approach has been announced as the Safe System approach.

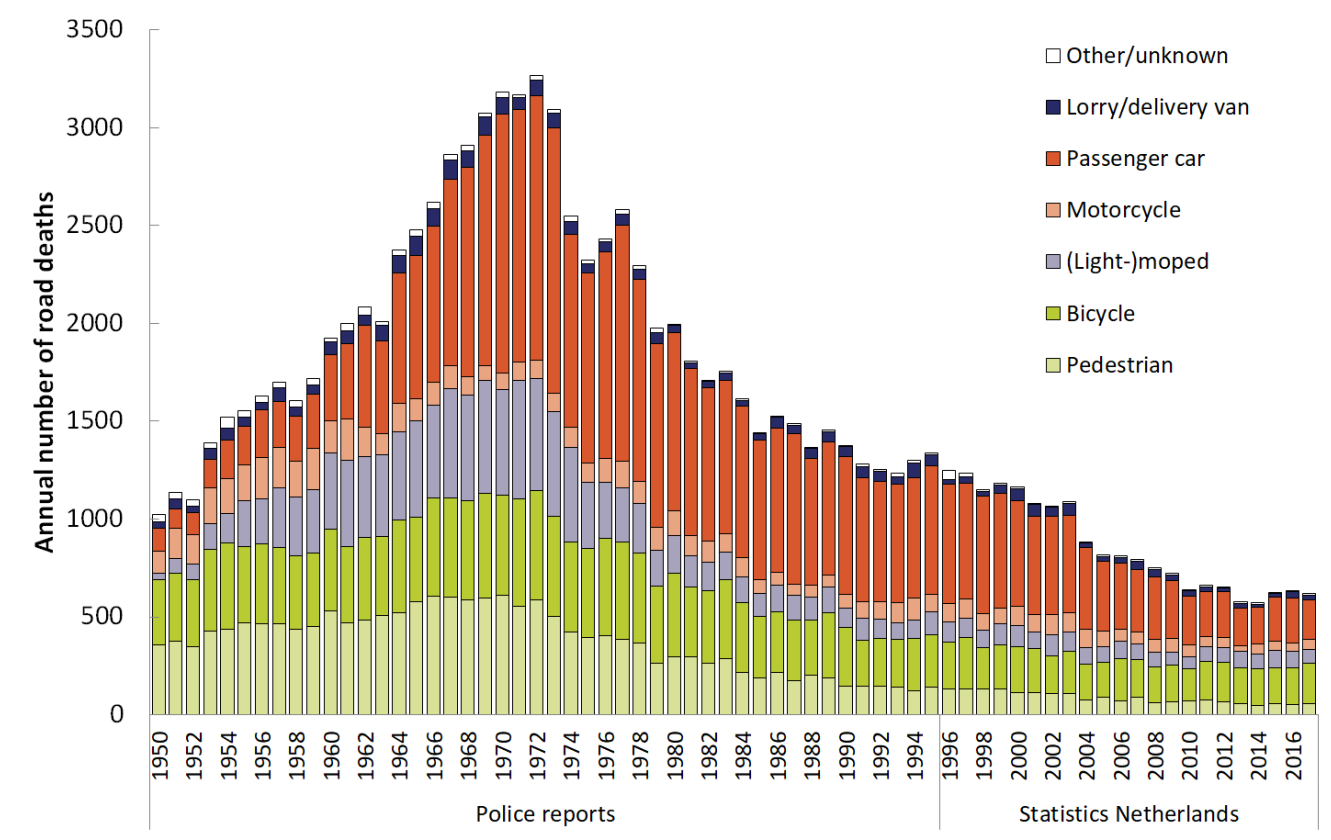

Fig. 1 Annual number of road traffic deaths The Netherlands. [1]

Nowadays the number of traffic fatalities in countries with emerging economies develops more or less similarly to that in Western Europe in the 1970ies. Fig. 2 gives an illustration for India with a number of fatalities of 150000 in 2015 . Like 50 years ago in The Netherlands safety belt use is promoted and enforcement strategies on speed and alcohol use develop. Gururaj and Gautham [2] give an extensive overview of the situation in India and about policies needed to reach improvements. And indeed casualty numbers after 2015 show a slight decline, although reliability still may be limited. Similar developments take place in many low and middle income countries, particularly in case of emerging economies.

In March 2010 the UN General Assembly proclaimed the Decade of Action for Road Safety. The Action intended to reach significant improvements in road safety and worked with a program based on 5 pillars: road safety management; safer roads and mobility; safer vehicles; safer road users; and post-crash response. 


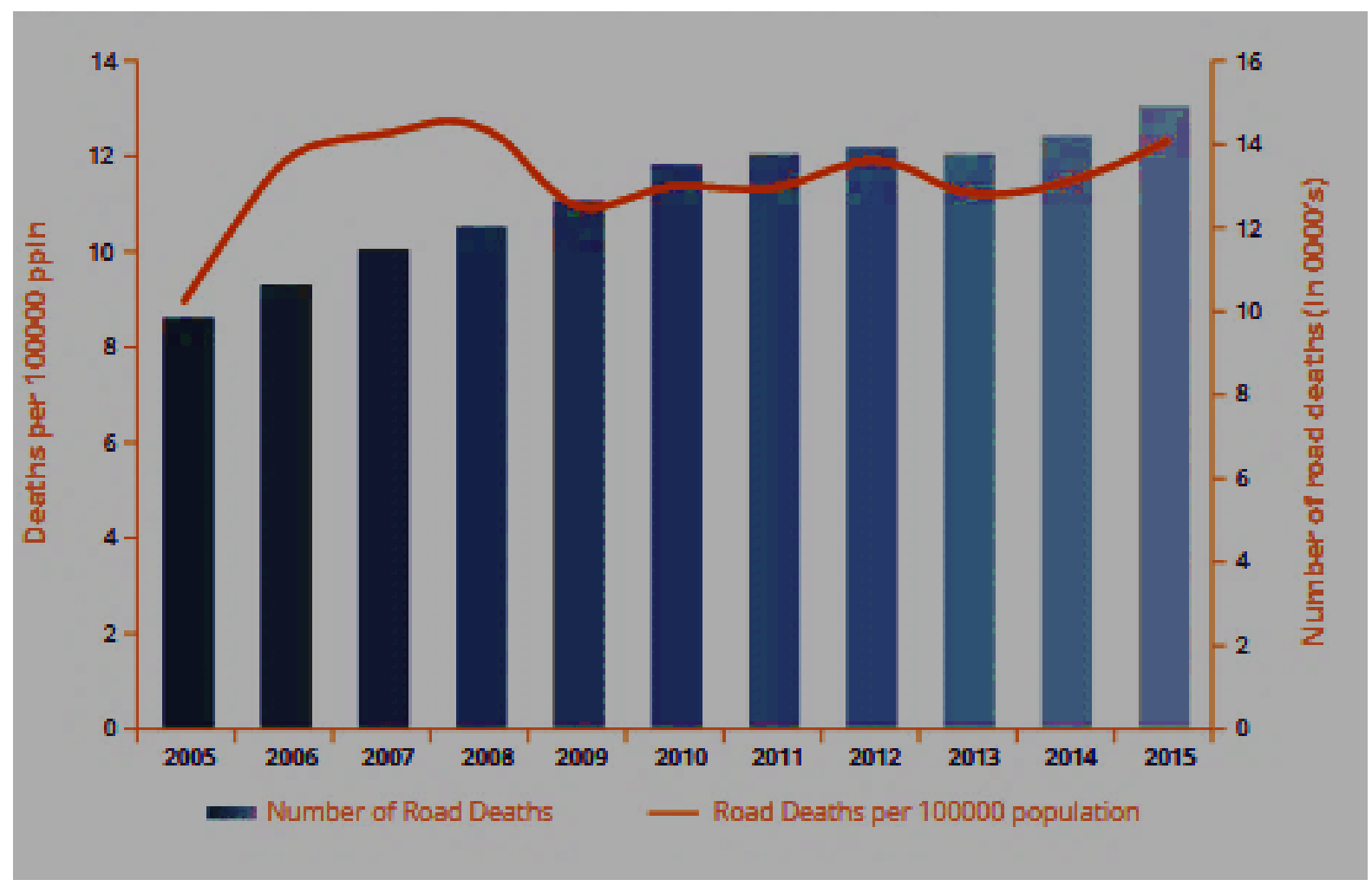

Fig. 2. Annual number of road traffic deaths in India. [2]

In addition the Brasilia Declaration on Road safety was signed in 2015. Countries agreed to halve road traffic deaths by the end of the decade - a key milestone within the new Sustainable Development Goal (SDG) target 3.6. The 2030 United Nations Agenda for Sustainable Development recognizes that road safety is a prerequisite to ensuring healthy lives, promoting well-being and making cities inclusive, safe, resilient and sustainable.

Now that the $1^{\text {st }}$ Decade of Action has reached 2020 some first positive results become visible. However at the $2 \mathrm{n}^{\mathrm{d}}$ Ministerial Conference on Road Safety in Stockholm (2020) the conclusion had to be drawn that the 2020 goals were by far not reached and that in fact the fatality numbers were still increasing. Fig. 3 shows the trend as illustrated in the WHO Global Status Report on road safety [3] in 2018 with a dramatic number of 1.35 million fatalities worldwide in 2016.

These number make clear that extra efforts are needed. Although the Safe System approach is advocated as the holy grail for definite improvements a systematic approach is lacking in many countries. Focusing on safer vehicles and roads as such will not lead to a safe traffic system. Also the question rises whether and how new visions on the use of connected traffic systems, vehicles, roads and users, anno 2020, may be made more effective in the battle for road safety. A fundamental question is whether on a relatively short notice the focus may shift to making the functional characteristics of vehicle support systems an integrated part of the safe system approach. Making use of existing, cheap technology may stimulate countries to develop policies towards a leapfrog strategy in order to improve their fatality numbers. This paper gives some thoughts into 
this direction. In future intelligent cars, trucks and buses should be able to understand roads and give guidance to the driver. This would not only make roads self-explaining but make the traffic system self-explaining as a whole. A safe system.

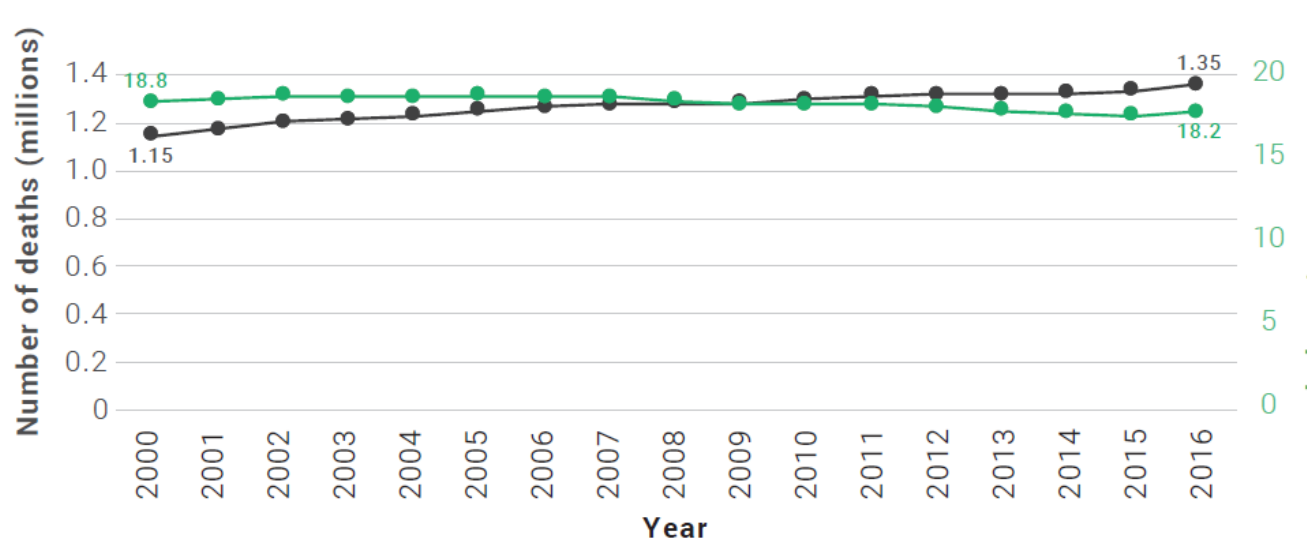

Number of deaths

Rate of death

Fig. 3 Number and rate of road traffic death per 100000 population worldwide 2000-2016. [3]

\section{Safe system approach}

In many western countries the safe system approach resulted in an immense improvement in traffic safety. Belle et al (2018) give a nice overview of the concept, its results and potential role in low and middle income countries [4]. The Dutch Sustainable Safety approach served as one of the building stones of the Safe System approach [5]. Both concepts are strongly related. Table 1 gives a description of the principles behind the Sustainable Safety concept.

Table 1. Description of the five Sustainable Safety principles. [5].

\begin{tabular}{|l|l|}
\hline Sustainable Safety Principle & Description \\
\hline Functionality of roads & $\begin{array}{l}\text { Monofunctionality of roads as either through roads, distributor roads, or access } \\
\text { roads in a hierarchically structured road network }\end{array}$ \\
\hline $\begin{array}{l}\text { Homogeneity of mass and/or speed and } \\
\text { direction }\end{array}$ & Equality of speed, direction, and mass at moderate and high speeds \\
\hline $\begin{array}{l}\text { Predictability of road course and road user } \\
\text { behaviour by a recognizable road design }\end{array}$ & $\begin{array}{l}\text { Road environment and road user behaviour that support road user expectations } \\
\text { through consistency and continuity of road design }\end{array}$ \\
\hline $\begin{array}{l}\text { Forgivingness of the environment and of } \\
\text { road users }\end{array}$ & $\begin{array}{l}\text { Injury limitation through a forgiving road environment and anticipation of road user } \\
\text { behaviour }\end{array}$ \\
\hline State awareness by the road user & Ability to assess one's capacity to handle the driving task \\
\hline
\end{tabular}


One of the fundamental premises behind the safe system approach is the notion that the road traffic system should take account of human limitations. The system is open to a variety of users of which children and elderly are a significant part. In addition lots of factors may be the reason for human failures. Fatigue, bad visibility, drivers being distracted and buses speeding because of tight time schedules. Unlike professional systems such as aviation, all of these aspects are to be included in an approach for a safe road traffic system. Human limitations therefore are to be considered as one of the basic characteristics behind the system. Analysis of human skills serves as an important tool for analysis of these limitations. To get a feeling about the traffic task it has proven useful to make a distinction between a number of task levels:

1. Modality level, e.g. deciding to travel by bus, car, bike, or as a pedestrian.

2. Network level: decisions about route choice and navigation;

3. Tactical level: decisions on lane changing, speeding, crossing, etc.

4. Operational level: vehicle control

A systems view on traffic behavior will take notice of the interactions between these different task levels. Development of a robust public transport system will serve as an important instrument to improve road safety. In future cities the clever mix of public and private transport will be leading in mobility development. Dividing the road network in a set of clearly defined road categories will guide route choice behavior. Such a network should also be based on a view on the functionality of roads. Roads may thus be categorized as either through roads, distributor roads, or access-roads in a hierarchically structured road network. Traffic signs and signals as well as road design characteristics will thus give guidance to road users in order to make optimal decisions.

The philosophy of Self Explaining Roads [6,7] served as one of the significant components of the Sustainable Safety recipe. The idea implies that the road network is designed in such a way that roads more or less naturally give control to human expectations and thus traffic behavior. Self-explaining roads naturally impose correct speeds and clearly indicate what type of traffic participants are to be expected and how interactions between the different types of participants should take place. Speed management is considered as significant factor in modern road design. On self-explaining roads this guidance process is more or less informal, but not without limiting conditions. Roundabouts do clearly limit speed and thus give guidance to the process of interaction with other road users on the particular intersection. Similarly speed humps and lifted intersections do limit speed in areas with a mix of cars and vulnerable road users. The concept of Self explaining roads has been advocated and applied on a broad scale, [8,9]

Ref. [4] emphasizes aspects like shared responsibility and the importance of planning, implementation, evaluation, and monitoring as key elements behind the safe systems approach and it is evident that all of these are of utmost importance, not only in western countries, but also in the low and middle income countries.

Nevertheless, for the arguments of the present paper it is important to note that the design principles behind the safe system approach are certainly not leading in today's infrastructure developments in low and middle income countries. Cities are getting larger and networks of roads are expanding in a chaotic manner. In many cases, existing through-roads in local 
communities are up-graded, resulting in heavy traffic loads, high speeds and mixed traffic on places, that are absolutely not suited for this kind of through-traffic.

The question therefore arises whether our expectations about the potential role of the Safe System Approach in its present shape on a worldwide scale are realistic. In many countries in Africa, Asia and Latin America roads and transport facilities may not be suited for a relatively quick transfer towards an orderly system and network. This may prevent a relatively quick 'leapfrog' towards the wished for situation with a reduction in the number of road traffic fatalities as intended. .

In order to reach that goal the safe system approach probably needs a new dimension. The challenge in road safety developments is to make a connection with new technologies, not only transport technologies, but also those in the area of communication (smartphones) and sustainable development (electric vehicles).

\section{Intelligent transport systems.}

Intelligent transport systems are on their way for about 30 years. The expectation has been and still is that in the course of time, subsequent levels of automation will appear ranging from no automation to driver assistance, to partial automation, to conditional automation and finally to full automation. [10, 11], see Table 2

Table 2. Levels of automation in road traffic $[10,11]$

No automation (level 0)

The full-time performance by the human driver of all aspects of the dynamic driving task, even when enhanced by warning or intervention systems

Driver assistance (level 1)

The driving mode-specific execution by a driver assistance system of either steering or acceleration/deceleration using information about driving conditions, with the expectation that the human driver performs all remaining dynamic aspects of the driving task

Partial automation (level 2)

The driving mode-specific execution by one or more driver assistance systems of both steering and acceleration/deceleration using information about driving conditions, with the expectation that the human driver performs all remaining dynamic aspects of the driving task

Conditional automation (level 3)

The driving mode-specific performance by an automated driving system of all dynamic aspects of the driving task, with the expectation that the human driver will respond appropriately to a request to intervene

High automation (level 4)

The driving mode-specific performance by an automated driving system of all dynamic aspects of the driving task, even if a human driver does not respond appropriately to a request to intervene

Full automation (level 5) 
The full-time performance by an automated driving system of all dynamic aspects of the driving task under all roadway and environmental conditions that can be managed by a human driver

The potential safety effects of intelligent transport systems has been analyzed and described extensively and thoroughly. Carsten and Tate (2005) illustrated that fatality numbers may be reduced by more than $50 \%$ by implementing intelligent speed adaptation. [12]. Wilmink et.al (2008) made an estimate about the safety effects of 25 intelligent vehicle safety systems, see Table 3.

[13]. Vaa et al. [14]. compared safety effects of different support systems, also indicating highest effects of speed adaptation systems. Kulmala (2010) developed a comprehensive framework for the safety assessment of intelligent transport systems. [15].

Table 3. Effects of 12 Intelligent Vehicle Safety System (IVSS) on the number of road fatalities in 25 EU member states in case of $100 \%$ fleet penetration by safety mechanism [10].

\begin{tabular}{|c|c|c|c|c|c|c|c|c|c|}
\hline \multirow[t]{2}{*}{ System } & \multicolumn{9}{|c|}{ Effects of IVSS on the number of road fatalities by safety mechanism (\%) } \\
\hline & 1 & 2 & 3 & 4 & 5 & 6 & 7 & 8 & 9 \\
\hline Electronic stability control ESC (corrected by author) & -21.1 & & +4.2 & +0.4 & & & & +0.5 & \\
\hline Full-speed range adapt. cruise control & -2.1 & & +0.9 & & & & & -0.2 & \\
\hline Emergency braking & -7.2 & & +0.3 & & & & & & \\
\hline Pre-crash protection for vulnerable road users & -2.5 & & +0.2 & & & & & & \\
\hline Lane change assistance & -2.3 & & +0.2 & & & +0.04 & & & \\
\hline Lane keeping Support & -17.7 & & +2.8 & & & & & & \\
\hline Night vision warning & -6.9 & & +3.5 & & & +0.7 & +0.3 & +0.3 & \\
\hline Drowsiness monitoring/warning & -7.9 & & +2.2 & & & +0.7 & +0.6 & +0.0 & \\
\hline Emergency call eCall & & & & & & & & & -5.8 \\
\hline Intersection safety support & -8.6 & & +5.0 & & & & & & \\
\hline Wireless local danger warning & -3.1 & & +0.2 & & -1.6 & & & & \\
\hline Speed alert & -5.5 & -1.0 & -1.0 & & -1.0 & & & -0.5 & \\
\hline $\begin{array}{l}\text { The safety mechanisms are: } \\
\text { (1) Direct in-vehicle modification of the driving task. } \\
\text { (2) Direct influence by roadside systems. } \\
\text { (3) Indirect modification of user behaviour. } \\
\text { (4) Indirect modification of non-user behaviour. } \\
\text { (5) Modification of interaction between users and non- } \\
\text { (6) Modification of exposure. } \\
\text { (7) Modification of modal choice. } \\
\text { (8) Modification of route choice. } \\
\text { (9) Modification of accident consequences only. }\end{array}$ & & & & & & & & & \\
\hline
\end{tabular}

Taken together most of the studies clearly point to the potential benefits of isolated intelligent support systems. Yet a more integrated vision about their role in the safe system concept is unclear and strongly underexposed.

The GIDS project [16, 17]. presented an early system philosophy, in which a number of isolated support systems are transformed into one integrated, communication system. As such GIDS made the connection between intelligent support systems and the safe system approach. GIDS provides driver support on all levels of the driving task, i.e. modality choice, navigation, anti-collision, speed adaptation, and active vehicle guidance. A dialog control system regulates the overall driver workload. GIDS connected car intelligence with local traffic circumstances. Although GIDS was developed in the pre-smartphone era, connections were also made with so-called applications, nowadays known as apps. 
Now that we live in the connected world of 2020 life is a lot easier, and the development of a GIDS like intelligent travel support system seems within reach. Cars, trucks and buses become more and more intelligent, i.e. sensing, thinking and explaining. Yes, also self-driving, but for the coming period, let 's say until 2050, the combination of driver support with partial automation seems achievable and by far most effective in terms of safety benefits. This perspective makes it rather disappointing that a vision about the role of connected mobility in the safety system context is still missing. Let's give it a try.

\section{Towards a safe system: vehicles that understand roads.}

Although the development of autonomous vehicles do get a lot of publicity, realistic expectations and predictions indicate that during the coming decades humans drive the car. Vision papers, like those by Price Waterhouse Cooper (2019) make a clear statement [18].

Various autonomous driving technologies already in use are designed to improve safety, such as rear-view cameras, automatic braking, adaptive cruise control, lane departure warning, etc. These options are increasingly implemented in autonomous vehicles of level 1 (function-specific) and 2 (combined function) and quickly become standard. .

The road to level 3 (limited self-driving) and level 4 (full self-driving) of autonomous driving is more difficult due to challenges that exist around high definition mapping, interaction with (and prediction of) human drivers and adaptation to changing infrastructures and circumstances. The complexity of this requires considerable investment and cooperation, the results of which will probably only be visible over time.

The authors of the WRI and Michelin vision papers [4, 10]. both show some hesitation about the potential safety benefits of connected mobility and intelligent transport systems. Leapfrogging toward a safe system is expected through strengthening institutions, frameworks, laws, regulations and guidelines. And of course this sort of developments are of crucial importance, together with making roads and vehicles safer through human oriented design. The safe system approach is a strong concept. No doubt.

However the safe system approach anno 2020 asks for a new vision about the link with concepts like connected mobility and the Internet of Things. Today cars, trucks and buses are connected with apps and smartphones, which brings us a myriad of options for personal assistance. All automobile manufacturers do deliver apps for Android and IoS systems They make their cars part of the personal network of their clients. The functionality of these Adaptive Driver Assistance Systems is diverse: traffic information, navigation, remote park information, speed assist, emergency support, driver alert, etc. The conceptual connection between this sort of personal assistance apps and the original GIDS concept is easily made.

Intelligent driver support apps like those in Polestar [19] serve as the dialog controller of the new smartphone oriented support system. The app will give support at the level of traffic modality ( what is faster, metro or car) and route (what is the safest and fastest route through the city network?) choice. And will also be connected to the settings of the lane guidance, speed 
adaptation and anti-collision system. Connecting the car to this personal assistant will ultimately make vision zero a realistic option in HIC.

Now that high level assistance systems are available the challenge is to use the basic version of this technology in LMICs as well. In-vehicle camera's give information about local traffic situation [20]. Smartphones are widely spread out worldwide as is the functionality of systems like Google maps. This makes the use of the elementary support functions (navigation, speed and cruise control, anti-collision) within reach, also in LMICs. New and older cars have to be connected to personal networks and related communication systems.

Relatively simple and cheap technologies may inform and guide drivers. Navigation systems may get the functionality of guiding drivers along safe routes. Speed can be influenced through enforcing and limiting, i.e. through the use of intelligent speed adaptation systems. Intersections may be controlled with the assistance of robust traffic sensors connected to the car. Vulnerable road users can be made visible in-car:

In other words: also in LMICs roads may become self-explaining - not only by their physical design characteristics - but also by guiding drivers and vulnerable road users through this sort of communication systems, related to route, speed and intersection control.

One of the crucial characteristics of a self-explaining road system is the categorization of the road network. Categories are linked to the functionality of roads: through roads, distributor roads and access roads. Connecting vehicles to road categories means that speed limits are available for intelligent speed adaptation, and optimal route guidance.

Anno 2030 connected mobility will be available in more or less definite form. Not only for HIC, but worldwide. The mixture of smartphones, navigation systems, intelligent speed adaption, anti-collision and lane keeping technology, based on smart sensors and satellite communication can be transformed into a relatively modest traffic guidance system, also in LMICs. Integration of these basic components into a driver support system would not bring us in the world of the self-driving car, but provide a tool to safely guide drivers in the complex myriad of roads. It will bring us in the world of guiding vehicles. Vehicles that are provided with a Generic User Interface for Driving Evolution. i.e. a GUIDE.

Such a GUIDE may have a broad functionality: it may help drivers optimize the driving task from the perspective of safety as well as sustainability. GUIDE may influence driving at the different levels of traffic behavior: it will provide guidance on public transport and parking facilities, traffic jams, helping people to choose their optimal and safe way of travelling. GUIDE will make drivers choose the safest route and speed, dependent on road quality, road categories and local traffic conditions GUIDE may influence speed through enforcing and limiting speed support systems, with intelligent speed adaptation as integrated element. By regulating drivers anticipation and expectations, GUIDE will make roads self-explaining - not only by their physical design characteristics - but also by providing in-car guidance for drivers. In future the functional characteristics of both cars and roads will be conceptualized into one integrated safe system, in 
which the user plays the central role. As such GUIDE may serve as the conceptual bridge between vehicle and roadway characteristics.

For the purpose of use in LMICs, such a GUIDE may be developed using relatively simple and cheap technologies, most of which are available. It can be argued that GUIDE brings us in the world of safe system approach 2.0. This development is necessary to give an acceleration towards zero fatalities in HICs and a breakthrough in road safety developments in LMICs.

\section{Final remarks}

The $2^{\text {nd }}$ Decade of Action for Road Safety 2021-2030 [21] as proclaimed by the United Nations General Assembly gives an important framework for safety interventions.. The Safe System approach is considered as the challenging framework for the actions needed. However, unfortunately the aspired goal of the $1^{\text {st }}$ Decade, i.e. a strong reduction in fatality numbers in the period until 2020, has not succeeded. Therefore this paper argues that for LMICs and particularly for emerging countries like India, Brazil, Nigeria, etc. the concept of safe systems approach should be upgraded. A safe system approach 2.0 is needed. The notion of sustainable roads should be integrated with that of vehicles giving guidance to drivers. The way to self-driving cars, should take a route via this concept, i.e. vehicles that guide drivers, both on self-explaining roads and on more or less unsafe roads. This approach will give a new dimension to the safe system approach. It would require policy makers, road designers, automobile manufacturers, traffic and mobile app industry to integrate 'traditional' issues like safe roads and safe vehicles towards a concept of safe traffic in which vehicles understand roads and guide drivers in behaving safely.

The safe system approach 2.0 based on functional integration of roads and vehicles will at the end not only be highly profitable for road safety in emerging countries. It will also give the ultimate solution for the remaining unsafety in Western Europe. The approach will give us a definite picture of a traffic world without accidents.

\section{References}

1. SWOV. Road deaths in the Netherlands, 2018, Road safety Research Institute SWOV Fact sheet, The Hague, The Netherlands.

2. Gururaj G, Gautham M S. Advancing Road Safety in India-Implementation is the Key, Bengaluru, National Institute of Mental Health \& Neuro Sciences; 2017. 60p. Publication Number:137.

3. WHO, Global status report on road safety 2018. Geneva: World Health Organization . Licence: CC BYNC

4. Welle, N., Sharpin, A.B. Adriazola-Steil, C., Batt, A., Alveano, S, Obelheiro, M., Tolga Imamoglu, C., Job, S., Shotten, M., and Bose, D., Sustainable and Safe: a vision and guidance for zero road deaths, 2018. World Resources Institute and Global Road Safety Facility, ISBN:978-1-56973-927-7 
5. SWOV, Advancing Sustainable Safety. Wegman, F.C.M., and Aarts, L.T., eds. 2006, The Hague, The Netherlands

6. Godthelp, J. Towards control in road traffic. (in Dutch) Verkeerskunde, 41, nr. 3. 1990 The Netherlands

7. Theeuwes, J. and Godthelp. J. Self-explaining roads. Safety Science, Volume 19, Issues 2-3, June 1995, Pages 217-225

8. Bekiaris, E., and Gaitanidou, E., Towards Forgiving and Self-Explanatory Roads. In: E. Bekiaris et al. (eds.), Infrastructure and Safety in a Collaborative World, DOI 10.1007/978-3-642-18372-0_2, 2011, Springer-Verlag Berlin Heidelberg, Germany.

9. Van Geem, C., Charman, S., Ahern, A., Anun, A., Sjögren, L., Pumberger, A., Grayson, G., and Helman. S, Speed adaptation control by Self explaining roads (SPACE). 16th Road Safety on Four Continents Conference Beijing, 2013, China 15-17

10. Michelin. Road safety and connected mobility. An overview report on the current status and implications of road safety and connected mobility. Michelin Challenge Bibendum. 2014 https: //community.Michelinchallenge bibendum. Com

11. Society of Automotive Engineers, https://www.sae.org/misc/pdfs/automated driving.pdf, 2018-06-15

12. Wilmink, I., Janssen, W., Jonkers, E., Malone, K., van Noort, M., Klunder, G., Rämä, P., Sihvola, N., Kulmala, R., Schirokoff, A., Lind, G., Benz, T., Peters, H., Schönebeck, S., Impact assessment of intelligent vehicle safety systems. eIMPACT Deliverable D4. July 2008

13. Kulmala, R. Ex-ante assessment of the safety effects of intelligent transport systems. Accident Analysis and Prevention, 42 (2010) 1359-1369

14. Carsten, O.M.J. and Tate, F.N. Intelligent speed adaptation: accident savings and cost-benefit analysis. Accident Analysis and Prevention, 37 (3). pp. 407-416. 2005, ISSN 0001-4575

15. Vaa, T., Assum, T. and Elvik, R. Driver support systems: Estimating road safety effects at varying levels of implementation. TØI report 1304/2014, Institute of Transport Economics, Oslo, Norway.

16. Godthelp, J. and Op de Beek, F. Driving with GIDS: Behavioural Interaction with the GIDS Architecture, Advanced Telematics in Road Transport, Proceedings of the DRIVE Conference, Brussels, 1991, p. $351-370$

17. Michon, J. A. (Ed.). Generic intelligent driver support. 1993, London: Taylor \& Francis; 255 pp

18. Price, Waterhouse, Cooper, https://www.pwc.nl/en/industries/automotive/the-future-of-mobility.html, 2019

19. Polestar owners manual : https://support.polestar.com/us/polestar-2/2021/article/Driver-support-systems/2020

20. IRAP Road that cars can read. European Road Assessment Programme, International Road Assessment Programme, and Road Safety Foundation, June 2018.

21. UNRSC, https://undocs.org/en/A/RES/74/299, 2020-08-31 